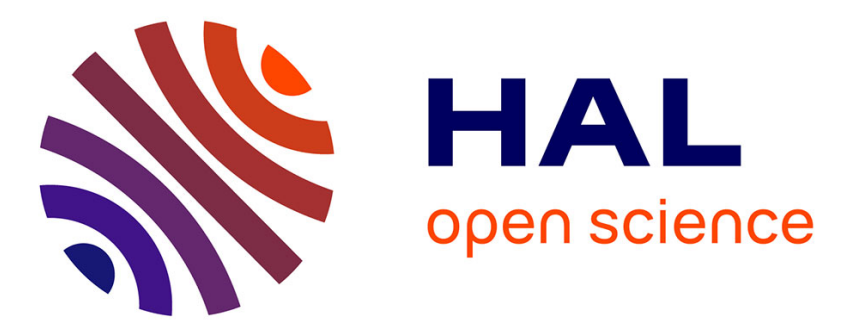

\title{
Coverage-extended cooperative geographic routing with optimal power allocation for wireless multihop networks
}

Syue-Ju Syue, Chin-Liang Wang, Vincent Gauthier, Pei-Shan Tsai

\section{To cite this version:}

Syue-Ju Syue, Chin-Liang Wang, Vincent Gauthier, Pei-Shan Tsai. Coverage-extended cooperative geographic routing with optimal power allocation for wireless multihop networks. VTC '12-Spring: IEEE 75th Vehicular Technology Conference, May 2012, Yokohama, Japan. pp.1-6, 10.1109/VETECS.2012.6240215 . hal-00718533

\section{HAL Id: hal-00718533 \\ https://hal.science/hal-00718533}

Submitted on 17 Jul 2012

HAL is a multi-disciplinary open access archive for the deposit and dissemination of scientific research documents, whether they are published or not. The documents may come from teaching and research institutions in France or abroad, or from public or private research centers.
L'archive ouverte pluridisciplinaire HAL, est destinée au dépôt et à la diffusion de documents scientifiques de niveau recherche, publiés ou non, émanant des établissements d'enseignement et de recherche français ou étrangers, des laboratoires publics ou privés. 


\title{
Coverage-Extended Cooperative Geographic Routing with Optimal Power Allocation for Wireless Multihop Networks
}

\author{
Syue-Ju Syue, Chin-Liang Wang, Vincent Gauthier*, and Pei-Shan Tsai \\ Institute of Communications Engineering, National Tsing Hua University, Hsinchu, Taiwan \\ Emails: d949619@oz.nthu.edu.tw, clwang@ee.nthu.edu.tw, s9864547@m98.nthu.edu.tw \\ * Lab. CNRS SAMOVAR UMR 5157, Telecom Sud Paris, Evry, France \\ Email: vincent.gauthier@telecom-sudparis.eu
}

\begin{abstract}
Cooperative geographic routing has emerged as an efficient, scalable routing paradigm that exploits the joint merit of cooperative diversity and position-aided localized operation to enhance the network performance. In terms of the hop efficiency, an existing scheme called Relay-Aware Cooperative Routing (RACR) has offered a cross-layer approach based on its quantitative study of how physical-layer cooperation leads to link-layer radio coverage extension, under the policy of equal power allocation (EPA) over the cooperating nodes. However, the effect of cooperation-based radio coverage extension dramatically varies with the power allocation policy in use. In this paper, we extend the RACR scheme by addressing a power-optimized cooperative geographic routing problem. With the joint optimization of relaying position and power allocation, the maximum cooperative transmission range is derived. We then, according to the result, present a modified cross-layer routing protocol taking into account the effectuation of optimal power allocation (OPA). Simulation results show that the proposed scheme effectively improves the coverage extension performance as well as reduces the average number of hops.
\end{abstract}

\section{INTRODUCTION}

Geographic routing [1]-[3], relying on the knowledge of geographic location information of nodes to make local route decisions, is a promising routing solution to the demand of developing efficient and scalable protocols in multihop wireless ad hoc networks. In recent years, with the significant advances of physical layer transmission techniques, cooperative communication for wireless networks has become an active research area due to its ability to create spatial diversity via node cooperation. There have been various cooperative diversity schemes in the literature [4]-[6]. However, most of them focus on the design of physical-layer cooperative relaying schemes, in which different issues such as signaling strategies, power allocation, relay selection, and bandwidth efficiency are taken into consideration to assess their physical layer performances.

In addition to the spatial diversity gain from cooperative communication, there is also a potential advantage in terms of coverage extension, which is associated with the link layer connectivity and, hence, affects the routing design and

This work was supported by the National Science Council of the Republic of China under Grants NSC 97-2221-E-007-009-MY3 performance at the network layer. However, this is ignored by most existing studies. There is very little insight into the impact of physical-layer cooperation on the networklayer routing design and performance. In [7], two heuristic routing algorithms called CTNCR and SNER are proposed to choose the minimum-power route with fixed transmission rate guaranteed. In [8], the authors demonstrated that the minimum energy cooperative path (MECP) routing problem is NP-complete, and then proposed a cooperative shortest path routing algorithm to achieve nearly the MECP. In [9], the authors proposed the Minimum Power Cooperative Routing (MPCR) algorithm, which constructs the minimum-power route with certain throughput guaranteed. In [10], the authors proposed the CoopGeo protocol, which provides an efficient approach to select the next hop as well as the optimal relay based on cross-layer designed geographic routing.

Most of the existing cooperative routing solutions are realized either by finding a route first in a traditional manner and then using cooperative transmission to enhance the link quality along the established route or by building the minimum-power route that applies a cooperation scheme to save the required transmission power. Nevertheless, these cooperative routing solutions do not take advantage of coverage extension of physical-layer node cooperation, since the cooperative route with coverage extension may be radically different from previous ones. Considering the demands for high efficiency and scalability in multihop routing as well as the potential benefit of coverage extension from cooperative transmission, in [11] we have investigated how the traditional non-cooperative geographic routing can be improved by incorporating the cooperative transmission into the design. Specifically, the previously proposed routing protocol, namely Relay-Aware Cooperative Routing (RACR), enables a forwarding node to make a local route decision depending on the geographic location of its relay while the relay is selected with the purpose of providing the maximum coverage extension toward the destination. However, the cooperative geographic routing scheme discussed in [11] is based on the use of equal power allocation. it can be expected that, with optimal power allocation, we can further improve the radio coverage extension of RACR. This can be 
clearly seen in [12] with an example of the comparison of the optimal power allocation and equal power allocation for the DF cooperative system. In that case, the DF scheme with optimal power allocation provides 2-dB SNR gain as compared to that with the equal power allocation and BPSK modulation. This motivates us to consider how to apply optimal power allocation to improve the radio coverage extension of RACR.

\section{SySTEM Model}

\section{A. Cooperation Strategy}

Our cooperation strategy follows those of [11], [13], using a modified version of the three-node decode-and-forward (DF) half-duplex signaling scheme as presented in [4]. The scheme consists of a sender $S$, a relay $R$, and a receiver $D$. In each hop, $S$ broadcasts its symbol to both $R$ and $D$ with transmit power $P_{1}$, and then $R$ decodes the received symbol and decides whether to relay it to $D$. It is assumed that $R$ achieves correct reception of the symbol as long as its received signal-to-noise ratio (SNR) exceeds a certain threshold. In this case, $R$ performs relaying with transmit power $P_{2}$; otherwise, it remains idle. Finally, $D$ combines the received symbols from $S$ and $R$ using the maximum ratio combining (MRC) technique [14], and performs the maximum likelihood (ML) detection. Throughout this paper, we assume the Rayleigh frequency-flat fading channels between any two nodes $i$ and $j$. The channel coefficient $h_{i, j}$ follows the distribution: $h_{i, j} \sim$ $C N\left(0, \sigma_{i, j}^{2}\right)$, where the channel gain variance $\sigma_{i, j}^{2}$ is modeled using a $\sigma_{i, j}^{2} \propto d_{i, j}^{-\alpha} \log$-distance path loss model [8], [15], where $\alpha$ is the path loss exponent and $d_{i, j}$ is the distance. We assume $h_{i, j}$ keeps constant over the symbol duration and may change from a symbol to another as an i.i.d. random process. In addition, the noise term is modeled as a zero-mean complex Gaussian random variable with variance $N_{0}$.

As analyzed in [12], the average SER performance of the above cooperation scheme using $M$-QAM modulation can be expressed as:

$$
P_{\mathrm{SER}}^{C}\left(\bar{\gamma}_{S, D}, \bar{\gamma}_{S, R}, \bar{\gamma}_{R, D}\right) \approx \frac{1}{b^{2} \bar{\gamma}_{S, D}}\left(\frac{A^{2}}{\bar{\gamma}_{S, R}}+\frac{B}{\bar{\gamma}_{R, D}}\right),
$$

where $\bar{\gamma}_{S, D}=P_{1} \sigma_{S, D}^{2} / N_{0}, \bar{\gamma}_{S, R}=P_{1} \sigma_{S, R}^{2} / N_{0}$ and $\bar{\gamma}_{R, D}=$ $P_{2} \sigma_{R, D}^{2} / N_{0}$ are the average received SNRs per symbol, $b=$ $3 / 2(M-1), A=(M-1) / 2 M+(1-1 / \sqrt{M})^{2} / \pi$, and $B=3(M-1) / 8 M+\left(1-1 / \sqrt{M}^{2} / \pi\right.$. Note that (1) is a tight approximation in a high SNR regime.

\section{B. Network Model}

Consider a wireless multihop ad hoc network that is modeled as a 2-dimensional graph $G=(V, E)$, where $V$ is the set of homogeneous nodes with $|V|=N$ and $E$ is the set of communication links between nodes. We consider a single session in the network, where data delivery may cross over multiple hops. Given a source-destination pair $(s, d)$, the other network nodes are ready to serve as intermediate nodes. An intermediate node has two roles-either a relay node used for cooperative relaying or a forwarding node used in the traditional multihop sense. In addition, two basic assumptions ${ }^{1}$ for the proposed protocol are made as follows: 1) every node is aware of its own geographic location, which can be accomplished by means of a localization technique such as [16] or a GPS (Global Positioning System) receiver [17]; 2) the location information of the destination can be obtained by the source via location service [18], [19]. Based on the philosophy of Greedy Forwarding, the goal is to make local route decisions for establishing a path from $s$ to $d$, along which each hop uses the power-optimized cooperation under an SERbased QoS (Quality-of-Service) constraint.

\section{SER-Based Radio Coverage}

In [11], we have defined the SER-based radio coverage to identify coverage extension from physical-layer cooperation. As a follow-up study of [11], we highlight some important definitions and properties as follows. Throughout this paper, we consider that the network graph $G=(V, E)$ is mapped into a 2-dimensional geographic plane, where every node $i \in V$ is characterized by its geographic location $\mathbf{x}_{i}$.

Definition 1: The SER-based radio coverage $\mathcal{R} \subset \mathbb{R}^{2}$ of a transmitting side $t$ is defined as a geographic area within which any receive node can meet certain SER requirement. Formally,

$$
\mathcal{R}=\left\{\mathbf{x} \in \mathbb{R}^{2} \mid P_{\text {SER }}\left(\mathbf{x}_{t}, \mathbf{x}\right) \leqslant \zeta_{0}\right\},
$$

where $\mathbf{x}_{t}$ denotes the location of the given transmitter $t, \mathbf{x}$ is a free point on $\mathbb{R}^{2}$ representing the location of the receiver, $P_{\mathrm{SER}}$ is the average SER at the receiver as a function of locations of both the transmitting side and receiver, and $\zeta_{0}$ is the required average SER at the receiver.

It is noted that: (i) the transmitting side $t$ can be a single transmit node using direct transmission or a set of cooperating nodes using cooperative transmission, (ii) given a communication model, the SER requirement $\zeta_{0}$ translates to a minimum received SNR throughout the radio coverage.

Based on Definition 1, the counterparts of direct and cooperative radio coverages are given as below.

Definition 2: The direct radio coverage of a transmit node $u$ with transmission power $P^{D}>0$ is defined as follows:

$$
\mathcal{R}^{D}(u)=\left\{\mathbf{x} \in \mathbb{R}^{2} \mid P_{\text {SER }}^{D}\left(\mathbf{x}_{u}, \mathbf{x}\right) \leqslant \zeta_{0}\right\} .
$$

For direct transmission, the radio coverage contour forms a circle with a radius $r^{D}$ around the sender because the path loss is the same at a uniform distance from the sender. In addition, based on the log-distance path loss model as described in Section II-A, the average received SNR can be expressed as a distance-dependent term:

$$
\bar{\gamma}\left(d_{i, j}\right)=\frac{P^{D} \sigma_{i, j}^{2}}{N_{0}}=\frac{P^{D}}{N_{0} d_{i, j}^{\alpha}} .
$$

\footnotetext{
${ }^{1}$ These two have been explicitly or implicitly used in the studies relevant to geographic routing.
} 
Therefore, (3) can be rewritten by:

$$
\begin{aligned}
\mathcal{R}^{D}(u) & =\left\{\mathbf{x} \in \mathbb{R}^{2} \mid\left\|\mathbf{x}-\mathbf{x}_{u}\right\| \leqslant\left(\frac{P^{D}}{N_{0} \bar{\gamma}_{0}}\right)^{\frac{1}{\alpha}}\right\} \\
& =\mathcal{D}\left(\mathbf{x}_{u}, r^{D}\right),
\end{aligned}
$$

where $\mathcal{D}\left(\mathbf{x}_{u}, r^{D}\right)$ is of a disk form with the center $\mathbf{x}_{u}$ and radius:

$$
r^{D}=\left(\frac{P^{D}}{N_{0} \bar{\gamma}_{0}}\right)^{\frac{1}{\alpha}},
$$

where $\bar{\gamma}_{0}$ is the required minimum received average SNR to meet an average SER requirement $\zeta_{0}$. In the case of direct transmission, given a required average SER $\zeta_{0}$ the corresponding $\bar{\gamma}_{0}$ can be obtained by taking an inverse operation of the following function:

$P_{\text {SER }}^{D}(\bar{\gamma})=2 K(1-g(\bar{\gamma}))+K^{2}\left(\frac{4}{\pi} g(\bar{\gamma}) \tan ^{-1}\left(g^{-1}(\bar{\gamma})\right)-1\right)$,

which is the average SER performance expression under the direct transmission with $M$-QAM modulation [20], where $K=1-1 / \sqrt{M}, g(\bar{\gamma})=(1+2(M-1) / 3 \bar{\gamma})^{-2}$, and $\bar{\gamma}=P^{D} \sigma^{2} / N_{0}$ is the average received SNR per symbol. In addition, we use the radius $r^{D}$ to specify neighbors within the direct coverage for a given node $u$. Similarly, we will also specify neighbors within the cooperative coverage by $r^{C}$.

Definition 3: Given a transmit node $u$ that cooperates with a given set of relays $V_{r}=\left\{r_{1}, r_{2}, \ldots, r_{N_{r}}\right\}$, the cooperative radio coverage of the transmit node $u$ with respect to the relay set $V_{r}$ is defined as:

$$
\begin{array}{r}
\mathcal{R}^{C}\left(u, V_{r}\right)=\left\{\mathbf{x} \in \mathbb{R}^{2} \mid P_{\mathrm{SER}}^{C}\left(\mathbf{x}_{u}, \mathbf{x}_{V_{r}}, \mathbf{x}\right) \leqslant \zeta_{0}, \mathbf{x}_{r_{i}} \in \mathcal{R}^{D},\right. \\
\text { for } \left.i=1,2, \ldots, N_{r}\right\} .
\end{array}
$$

Here we note that the cooperating relays are confined to the direct radio coverage of node $u$. In other words, the cooperating relays have to be one-hop neighbors of node $u$.

Definition 4: In the cooperative mode with a source node $u$ and a set of relays $V_{r}$, for a given destination node $v \in \mathcal{R}^{C}(u)$, the Euclidean distance between nodes $u$ and $v$, denoted by

$$
r^{C}(u, v)=\left\|\mathbf{x}_{v}-\mathbf{x}_{u}\right\|,
$$

is called the cooperative transmission distance between the source $u$ and destination $v$.

Definition 5: Consider a transmit node $u$ with a set of relays $V_{r}=\left\{r_{1}, r_{2}, \ldots, r_{N_{r}}\right\}$ that can be located at any places within $\mathcal{R}^{D}(u)$, the maximum cooperative radio coverage of the transmit node $u$ is defined as:

$$
\begin{array}{r}
\mathcal{R}_{\max }^{C}(u)=\left\{\mathbf{x} \in \mathbb{R}^{2} \mid P_{\mathrm{SER}}^{C}\left(\mathbf{x}_{u}, \mathbf{x}_{V_{r}}, \mathbf{x}\right) \leqslant \zeta_{0}, \forall \mathbf{x}_{r_{i}} \in \mathcal{R}^{D},\right. \\
\text { for } \left.i=1,2, \ldots, N_{r}\right\} .
\end{array}
$$

By definitions, it can be seen that: (i) $\mathcal{R}^{C}\left(u, V_{r}\right)$ is a subset of $\mathcal{R}_{\max }^{C}(u)$ and (ii) $\mathcal{R}_{\max }^{C}(u)$ is a disk centered at the location of the sender $u$ due to the symmetry of path loss in space:

$$
\begin{aligned}
\mathcal{R}_{\max }^{C}(u) & =\left\{\mathbf{x} \in \mathbb{R}^{2} \mid\left\|\mathbf{x}-\mathbf{x}_{u}\right\| \leqslant r_{\max }^{C}\right\} \\
& =\mathcal{D}\left(\mathbf{x}_{u}, r_{\text {max }}^{C}\right),
\end{aligned}
$$

where $r_{\max }^{C}$ is the maximum cooperative transmission distance of the source node $u$. In the next section, we give derivations to find the maximum cooperative transmission distance with the use of OPA.

\section{Analysis of Power-Optimized Cooperative RADIO COVERAGE}

Consider the three-node DF cooperation model $(s, r, f)$ with $M$-QAM modulation under the OPA policy. Our goal is to derive the optimal powers $P_{1}^{*}$ and $P_{2}^{*}$ as well as the corresponding maximum cooperative radio coverage radius $r_{\max }^{C}$.

By using (4), the SER expression in (1) can be rewritten in relation to the distance-dependent arguments by:

$$
\begin{aligned}
P_{\mathrm{SER}}^{C}\left(\bar{\gamma}_{s, f}, \bar{\gamma}_{s, r}, \bar{\gamma}_{r, f}\right) & \approx \frac{1}{b^{2} \bar{\gamma}_{s, f}}\left(\frac{A^{2}}{\bar{\gamma}_{s, r}}+\frac{B}{\bar{\gamma}_{r, f}}\right) \\
& =\frac{N_{0}^{2}}{b^{2}} d_{s, f}^{\alpha}\left(\frac{A^{2} d_{s, r}^{\alpha}}{P_{1}^{2}}+\frac{B d_{r, f}^{\alpha}}{P_{2}}\right) \\
& =P_{\mathrm{SER}}^{C}\left(d_{s, f}, d_{s, r}, d_{r, f}\right) .
\end{aligned}
$$

Then $d_{s, f}$ can be expressed as:

$$
d_{s, f}=\left(\frac{b^{2} P_{\mathrm{SRR}}^{C}}{N_{0}^{2}\left(\frac{A^{2} d_{s, r}^{\alpha}}{P_{1}^{2}}+\frac{B d_{r, f}^{\alpha}}{P_{2}}\right)}\right)^{\frac{1}{\alpha}} .
$$

From Definition $4, d_{s, f}$ in (13) represents the cooperative transmission distance.

Since the source node $s$ is fixed whereas the relay node $r$ is a free point that causes different cooperative transmission distances along the direction of $\left(\mathbf{x}_{r}-\mathbf{x}_{s}\right)$ depending on its location, by letting $d_{s, f}=r^{C}\left(\mathbf{x}_{r}\right)$ and $P_{\mathrm{SER}}^{C}=\zeta_{0}$ (denoting the required average SER) we can formulate the following function from (13):

$$
r^{C}\left(\mathbf{x}_{r}, P_{1}, P_{2}\right)=\left(\frac{b^{2} \zeta_{0}}{N_{0}^{2}\left(\frac{A^{2}\left\|\mathbf{x}_{r}-\mathbf{x}_{s}\right\|^{\alpha}}{P_{1}^{2}}+\frac{B\left\|\mathbf{x}_{r}-\mathbf{x}_{f}\right\|^{\alpha}}{P_{2}}\right)}\right)^{\frac{1}{\alpha}} .
$$

Firstly we focus on the linear network case. Let $k$ be a normalized position of the relay node, given by:

$$
k=\frac{d_{s, r}}{d_{s, f}}=\frac{\left\|\mathbf{x}_{r}-\mathbf{x}_{s}\right\|}{\left\|\mathbf{x}_{f}-\mathbf{x}_{s}\right\|} .
$$

We then have

$$
\begin{aligned}
& d_{s, r}=k \cdot d_{s, f}=k \cdot r^{C}\left(\mathbf{x}_{r}, P_{1}, P_{2}\right) ; \\
& d_{r, f}=(1-k) d_{s, f}=(1-k) \cdot r^{C}\left(\mathbf{x}_{r}, P_{1}, P_{2}\right) .
\end{aligned}
$$

By substituting (16) into (14), we rewrite (14) as:

$$
\begin{aligned}
& r^{C}\left(\mathbf{x}_{r}, P_{1}, P_{2}\right)= \\
& \left(\frac{b^{2} \zeta_{0}}{N_{0}^{2}\left(\frac{A^{2} k^{\alpha}\left(r^{C}\left(\mathbf{x}_{r}\right)\right)^{\alpha}}{P_{1}^{2}}+\frac{B(1-k)^{\alpha}\left(r^{C}\left(\mathbf{x}_{r}\right)\right)^{\alpha}}{P_{2}}\right)}\right)^{\frac{1}{\alpha}} .
\end{aligned}
$$


Then, for ease of tracing we place the source node $s$ at the origin on $\mathbb{R}^{2}$, so (17) becomes:

$$
r^{C}\left(k, P_{1}, P_{2}\right)=\left(\frac{b^{2} \zeta_{0}}{N_{0}^{2}\left(\frac{A^{2} k^{\alpha}}{P_{1}^{2}}+\frac{B(1-k)^{\alpha}}{P_{2}}\right)}\right)^{\frac{1}{2 \alpha}} .
$$

Now our goal is to solve the following optimization problem for $r_{\max }^{C}$ :

$$
\begin{array}{ll}
\text { maximize } & r^{C}\left(k, P_{1}, P_{2}\right) \\
\text { subject to } & 0<k<1 \\
& P_{1}+P_{2}=P
\end{array}
$$

which is to find the optimal normalized position of the relay $k^{*}$ and the optimal power allocation $P_{1}^{*}$ and $P_{2}^{*}$ that provides the maximum cooperative transmission distance.

Since both the $P_{1}^{*}$ and $P_{2}^{*}$ are $k$-dependent (i.e., given a relay's position $k, P_{1}$ and $P_{2}$ can be optimized with respect to $k$ ), (19) becomes:

$$
\begin{array}{ll}
\text { maximize } & r^{C}\left(k, P_{1}^{*}(k), P_{2}^{*}(k)\right) \\
\text { subject to } & 0<k<1
\end{array}
$$

where, by invoking [12], $P_{1}^{*}(k)$ and $P_{2}^{*}(k)$ can be obtained by:

$$
\begin{aligned}
& P_{1}^{*}(k)=\frac{k^{-\alpha / 2}+\sqrt{k^{-\alpha}+8\left(A^{2} / B\right)(1-k)^{-\alpha}}}{3 k^{-\alpha / 2}+\sqrt{k^{-\alpha}+8\left(A^{2} / B\right)(1-k)^{-\alpha}}} P ; \\
& P_{2}^{*}(k)=\frac{\sqrt{2 k^{-\alpha / 2}}}{3 k^{-\alpha / 2}+\sqrt{k^{-\alpha}+8\left(A^{2} / B\right)(1-k)^{-\alpha}}} P .
\end{aligned}
$$

Now we aim to solve the optimization problem in (20). It has been proved in [11] that the following two optimization problems are equivalent under the same power allocation policy:

- Problem I: Given a source-destination pair, find the optimal normalized position of the relay to minimize the average SER at the destination.

- Problem II: Given an average SER performance requirement, find the optimal normalized position of the relay to maximize the cooperative transmission distance.

In our case it implies:

$$
\max _{k} r^{C}\left(k, P_{1}^{*}(k), P_{2}^{*}(k)\right) \equiv \min _{k} P_{\text {SER }}^{C}\left(k, P_{1}^{*}(k), P_{2}^{*}(k)\right) .
$$

Furthermore, the Problem I using EPA has been addressed in [15] and can be further expressed as:

$$
\begin{array}{ll}
\text { minimize } & A^{2} k^{\alpha}+B(1-k)^{\alpha} \\
\text { subject to } & 0<k<1,
\end{array}
$$

which, as introduced in [21], is an $\alpha$-order placement problem in a convex form and thus can be solved efficiently. Specially, for the path loss exponent $\alpha=2$, the solution has the closed form of $k^{*}=\frac{B}{A^{2}+B}$.

For the planar network case, [15] has also shown that the optimal position of the relay (regarding the Problem I) is on the line segment between the source-destination pair while approaching toward the middle point of them with increasing $\alpha$. Thus, according to (22), the above result still holds for this proposition (or, simply, the Problem II).

\section{Cross-Layer Protocol Design}

\section{A. Basic Idea}

The proposed cooperative routing protocol is based on a cross-layer design, which involves the relay selection and forwarder selection alike and can be viewed as a beaconless geographic routing scheme such as [3], [13]. The protocol takes advantage of coverage extension based on three-node cooperation to select intermediate nodes in a geographically greedy manner. As such, the required number of hops for given a source-destination pair can be reduced as compared to the traditional non-cooperative geographic routing. The radio coverage formulas for direct and cooperative transmission schemes, as well as the optimal positions for relaying and forwarding, are employed in this protocol.

Conceptually, the protocol is realized through a two-phase selection process. The first phase selects the best relay such that the power-optimized source-relay pair provides the maximum coverage extension toward the destination, whereas the second phase selects the forwarder (with respect to the already selected relay) for offering the largest progress toward the destination. Both the relay and forwarder selections are based on a distributed contention process without periodic exchanges of control messages (i.e., beacons) for acquiring neighbors' location information. During a contention process, candidate nodes compete for being a relay or a forwarder by setting contention timers that are relevant to their geographic locations.

\section{B. Contention-Based Relay and Forward Selections}

Since the best relay should be as close to the optimal relaying position $\mathbf{x}_{r}^{*}$ as possible, each candidate's timer value must be proportional to the distance between itself and $\mathbf{x}_{r}^{*}$. We map this distance into a normalized relay selection metric $\mathcal{M}_{r} \in[0,1]$ as given by:

$$
\mathcal{M}_{r}=\frac{\left\|\mathbf{x}_{r_{i}}-\mathbf{x}_{r}^{*}\right\|}{r^{D}+\left\|\mathbf{x}_{s}-\mathbf{x}_{r}^{*}\right\|},
$$

where $\mathbf{x}_{r_{i}}$ denotes the location of the candidate $r_{i}$ and the denominator represents the farthest distance between a possible candidate's and optimal relaying positions. Finally, we set the relay-selection contention timer for each candidate by

$$
T_{\text {relay }}=\frac{\left(N_{r}-1\right)}{N_{r}} T_{\max } \times \mathcal{M}_{r}+\operatorname{rand}\left(\frac{T_{\max }}{N_{r}}\right),
$$

where $N_{r}$ is the number of groups to be divided in the relaying area and $\operatorname{rand}(x)$ is a function obtaining a random value between 0 and $x$ to reduce the collision probability.

To select the forwarder with the largest progress over the cooperative radio coverage toward the destination, we set each candidate's contention timer to be proportional to the distance between its and the optimal forwarding positions. To do so, we first define a projection point $\mathbf{x}_{m_{i}}$ from the selected relay $\mathbf{x}_{r_{i}}$ 
onto the source-destination line using a trigonometric function. Given that the coordinates $\mathbf{x}_{s}, \mathbf{x}_{r}^{*}$, and $\mathbf{x}_{d}$ are known by each current node and that each possible relay knows its own $\mathbf{x}_{m_{i}}$ position, each candidate forwarder can derive the optimal forwarding position $\mathbf{x}_{f}^{*}$ and set its corresponding timer $T_{f w d}$. We define the forwarder selection metric $\mathcal{M}_{f}$ as:

$$
\mathcal{M}_{f}=\frac{\left\|\mathbf{x}_{f_{i}}-\mathbf{x}_{f}^{*}\right\|}{\sqrt{\left(r^{D}\right)^{2}+\left\|\mathbf{x}_{m_{i}}-\mathbf{x}_{f}^{*}\right\|^{2}}}
$$

where $\left\|\mathbf{x}_{m_{i}}-\mathbf{x}_{f}^{*}\right\|=r_{\max }^{C}-\left\|\mathbf{x}_{s}-\mathbf{x}_{m_{i}}\right\|$. Finally, we set the forwarder-selection contention timer for each candidate by:

$$
T_{f w d}=\frac{\left(N_{f}-1\right)}{N_{f}} T_{\max } \times \mathcal{M}_{f}+\operatorname{rand}\left(\frac{T_{\max }}{N_{f}}\right),
$$

where $N_{f}$ is the number of groups to be divided in the forwarding area.

\section{Protocol Description}

First, the source checks whether the channel is free during a predefined time interval before starting the transmission. If the channel is free, the source broadcasts CTS to its neighbors. Those neighbors within the maximum radio coverage decode the CTS message, including the direct transmission range and cooperative range. Second, the nodes which can decode CTS correctly set their timer to compete for becoming the relay. The winning relay (whose timer expires first) calculates $P_{1}^{*}$ and $P_{2}^{*}$ and sends the RTS that contains the information of $P_{1}^{*}$ to the source node, and then the source sends the data message. The other candidates overhearing the RTS or data message suppress their timers. Besides, the neighbors within the cooperative radio coverage store the data message form the source node. Third, the winning relay receives the data from the source, and then retransmits to its neighbors within the cooperative radio coverage. The source considers the overhearing data message as a passive acknowledgement (ACK), and stops its action. Forth, the candidate forwarders that can correctly perform the maximum ratio combining participate in the forwarder selection process by setting their timers. Fifth, once the winning forwarder comes out, it broadcasts the ACK to the relay node to indicate its correct reception. The other candidates overhearing the ACK cancel their timers and discard the data message. Finally, the forwarder becomes the current node and re-executes the same steps until the data message reaches the destination.

\section{Performance Evaluation}

In this section, we give simulation results and compare the performance of proposed the proposed scheme with the related protocol RACR. We assume that the channel gain variances of Rayleigh fading channels between any two nodes follow $\sigma_{i, j}^{2} \propto d_{i, j}^{-\alpha}$, where $d_{i, j}$ is the Euclidean distance between nodes $i$ and $j$ and $\alpha$ is the path loss exponent. Here, we set: $\alpha=4$, the average noise power $N_{0}=-70 \mathrm{dBm}$, the total transmit power of each cooperating pair $P=13.98 \mathrm{dBm}$, the average SER performance requirement $\zeta_{0}=10^{-2}$, and modulation type 4-QAM.

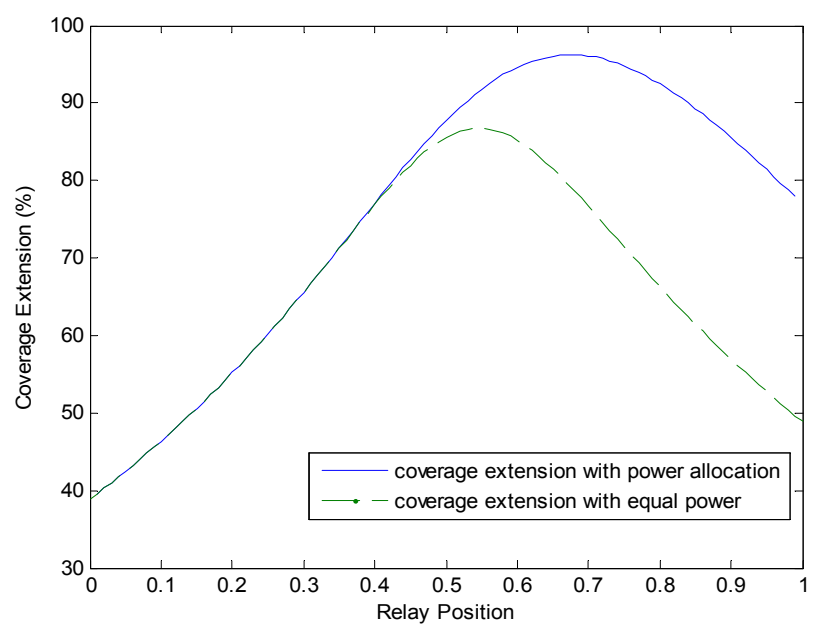

Fig. 1: Coverage extension comparison for RACR with EPA and OPA.

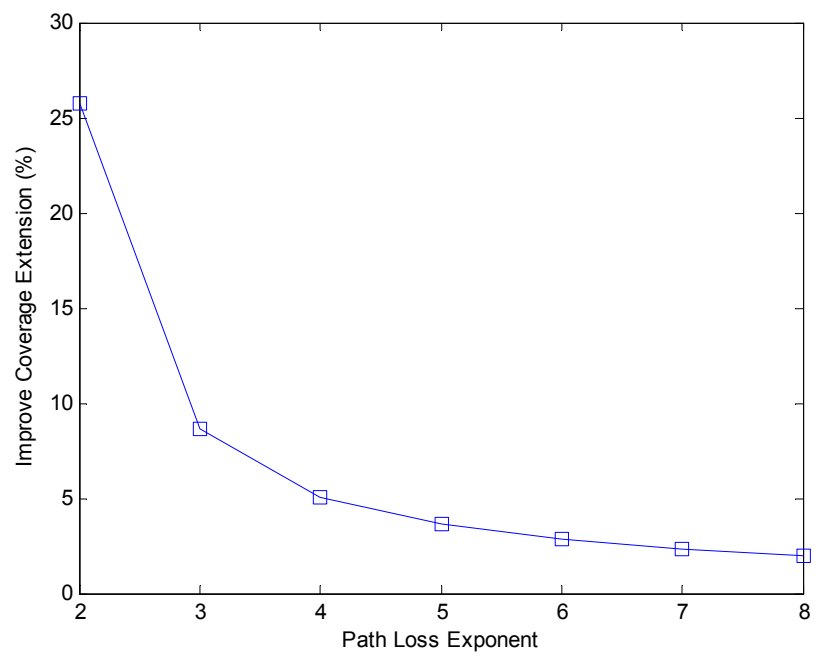

Fig. 2: Coverage extensibility of RACR-OPA over RACR-EPA.

First, we give the theoretical results to examine the coverage extension from the three-node cooperation model using EPA and OPA, respectively, as compared to the direct transmission. Fig. 1 depicts the percentage of increment of radio coverage with varying the relay's position between the source $(0,0)$ and the destination $(1,0)$ (normalized scale). It shows that the best relaying position for RACR is $(0.55,0)$, providing an about $85 \%$ coverage extension toward the destination; the other one, power-optimized RACR, indicates that the best relaying position is on $(0.69,0)$, providing an about $94 \%$ coverage extension toward the destination.

Next, we compare the coverage extensibility of RACR with OPA (RACR-OPA) as compared to the RACR with EPA (RACR-EPA) under different path loss exponents. Fig. 2 shows that the RACR-OPA outperforms significantly the RACREPA at low path loss exponents while their performance gap becomes smaller and smaller with increasing the path loss 


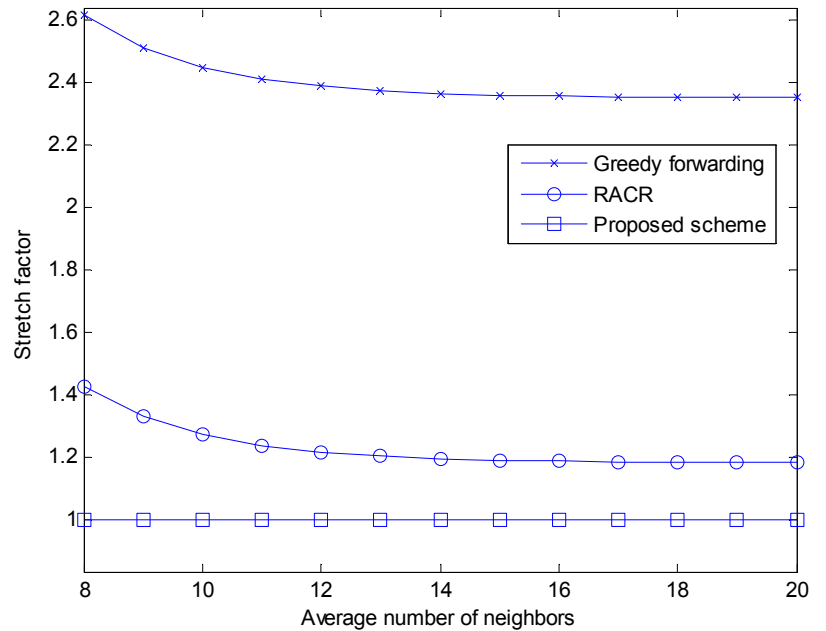

Fig. 3: The stretch factor versus the average number of neighbors.

exponent. This can be explained from the optimal power allocation result as given in (21). The optimal transmit powers $P_{1}^{*}$ and $P_{2}^{*}$ go to $P / 2$ (like the way RACR-EPA does) as the path loss exponent is sufficiently large.

Next we give the numerical results to evaluate the routing performance of the proposed protocol. We generate 100 network topologies where nodes are randomly deployed within a $1000 \times 1000 \mathrm{~m}^{2}$ field. For each topology, we randomly select 750 source-destination pairs. The simulation results are averaged over the $100 \times 750$ simulation runs. To demonstrate how the proposed RACR-OPA improves the RACR-EPA while the non-cooperative geographic greedy routing (GF) serves as a benchmark, we consider the energy efficiency by measuring their numbers of hops required. As set in the previous simulation, we restrict each hop-whatever transmission modes may be-has the same total transmit power. Thus, the performance metric of the path length (i.e., the number of hops) translates to energy efficiency. Fig. 3 shows the performance of the stretch factor $^{2}$ versus the average number of neighbors. It can be seen that the RACR-OPA protocol outperforms better than the RACR-EPA due to the effect of power-optimized cooperation. Thus, we demonstrate that the proposed RACR-OPA protocol is more energy/hop efficient than the existing RACR protocol.

\section{CONCLUSIONS}

In this paper, with the focus on the coverage-extended cooperative geographic routing [11], we have proposed an improved scheme based on optimal power allocation to enhance the radio coverage. We gain the potential advantage of the physical-layer cooperation in terms of radio coverage extension. For a sender cooperating with its optimal relay under the optimal power allocation strategy, the maximum cooperative transmission range was derived, proving the radio coverage improvement of optimal power allocation. To gain the maximum coverage extension toward the destination, we

\footnotetext{
2 The stretch factor is defined as the path length ratio.
}

first select the best relay that provids the maximum cooperative transmission distance, and then select the best forwarder whose geographic position is closest to the destination. The proposed protocol has been demonstrated by simulations to achieve higher hop efficiency than the existing RACR protocol.

\section{REFERENCES}

[1] I. Stojmenovic, "Position-based routing in ad hoc network," IEEE Commun. Mag., vol. 40, no. 7, pp. 128-134, July 2002.

[2] B. Karp and H. T. Kung, "GPSR: greedy perimeter stateless routing for wireless networks," in Proc. 4th ACM/IEEE Int'l. Conf. Mobile Computing and Networking (MobiCom 2000), Boston, MA, USA, Aug. 2000, pp. 243-254.

[3] J. A. Sanchez, P. M. Ruiz, and R. Marin-Perez, "Beacon-less geographic routing made practical: challenges, design guidelines, and protocols," IEEE Commun. Mag., vol. 47, no. 8, pp. 85-91, Aug. 2009.

[4] J. N. Laneman, D. N. C. Tse, and G. W. Wornell, "Cooperative diversity in wireless networks: efficient protocols and outage behavior," IEEE Trans. Inf. Theory, vol. 50, no. 12, pp. 3062-3080, Dec. 2004.

[5] C.-L. Wang and S.-J. Syue, "An efficient relay selection protocol in cooperative wireless sensor networks," in Proc. 2009 IEEE Wireless Commun. and Networking Conf., Budapest, Hungary, April 2009.

[6] K. Vardhe, D. Reynolds, and B. Woerner, "Joint power allocation and relay selection for multiuser cooperative communication," IEEE Trans. Wireless Commun., vol. 9, no. 4, pp. 1255-1260, Apr. 2010.

[7] Z. Yang and A. Host-Madsen, "Routing and power allocation in asynchronous gaussian multiple-relay channels," EURASIP J. Wireless Commun. Netw., vol. 44, pp. 181-217, Jan. 2006.

[8] F. Li, K. Wu, and A. Lippman, "Energy-efficient cooperative routing in multi-hop wireless ad hoc networks," in Proc. IEEE International Performance, Computing, and Communications Conference (IPCCC 2006), April 2006, pp. 215-222.

[9] A. Ibrahim, Z. Han, and K. Liu, "Distributed energy-efficient cooperative routing in wireless networks," IEEE Trans. Wireless Commun., vol. 7, no. 10 , pp. 3930-3941, Oct. 2008

[10] T. Aguilar, M. C. Ghedira, S.-J. Syue, V. Gauthier, H. Afifi, and C.L. Wang, "A cross-layer design based on geographic information for cooperative wireless networks," in Proc. 2010 IEEE Vehic. Tech. Conf. (VTC 2010-Spring), Taipei, Taiwan, May 2010, pp. 1-5.

[11] S.-J. Syue, C.-L. Wang, T. Aguilar, V. Gauthier, and H. Afifi, "Cooperative geographic routing with radio coverage extension for SERconstrained wireless relay networks," to appear, IEEE J. Sel. Areas Commun.

[12] A. K. Sadek, W. Su, and K. J. R. Liu, "Cooperative communication protocols in wireless networks: performance analysis and optimum power allocation," Wirel. Pers. Commun., vol. 44, pp. 181-217, Jan. 2008.

[13] T. Aguilar, S.-J. Syue, V. Gauthier, H. Afifi, and C.-L. Wang, "CoopGeo: A beaconless geographic cross-layer protocol for cooperative wireless ad hoc networks," IEEE Trans. Wireless Commun., vol. 10, no. 8, pp. 2554-2565, Aug. 2011.

[14] D. Brennan, "Linear diversity combining techniques," Proc. IEEE, vol. 91, no. 2, pp. 331-356, Feb. 2003.

[15] C.-L. Wang and S.-J. Syue, "A geographic-based approach to relay selection for wireless ad hoc relay networks," in Proc. 2009 IEEE Vehic. Tech. Conf. (VTC 2009-Spring), Barcelona, Spain, April 2009.

[16] N. Patwari, I. Hero, A. O., M. Perkins, N. S. Correal, and R. J. O'Dea, "Relative location estimation in wireless sensor networks," IEEE Trans. Signal Process., vol. 51, no. 8, pp. 2137-2148, Aug. 2003.

[17] E. D. Kaplan and C. J. Hegarty, GPS: Principles and Applications. MA: Artech House, 2006.

[18] T. Camp, J. Boleng, and L. Wilcox, "Location information services in mobile ad hoc networks," in Proc. 2002 IEEE Int. Conf. Commun. (ICC 2002), New York, NY, USA, Apr. 2002, pp. 3318-3324.

[19] E. Kuiper and S. Nadjm-Tehrani, "Geographical routing with location service in intermittently connected MANETs," IEEE Trans. Veh. Technol., vol. 60, no. 2, pp. 592-604, Feb. 2011.

[20] M. Simon and M. Alouini, "A unified approach to the performance analysis of digital communication over generalized fading channels," Proc. IEEE, vol. 86, no. 9, pp. 1860-1877, Sept. 1998.

[21] S. Boyd and L. Vandenberghe, Convex Optimization. Cambridge: Cambridge University Press, 2004. 